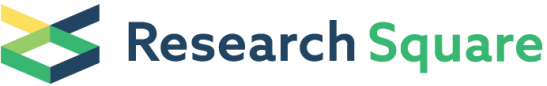 \\ Preprints are preliminary reports that have not undergone peer review. \\ They should not be considered conclusive, used to inform clinical practice, or referenced by the media as validated information.
}

\section{Four-dimensional hydrogel-in-hydrogel bioprinting for the spatiotemporal control of organoid and organotypic cultures}

Nicola Elvassore ( $\nabla$ nicola.elvassore@unipd.it)

University of Padua https://orcid.org/0000-0002-7029-6287

Anna Urciuolo

University of Padova https://orcid.org/0000-0001-7571-9747

Giovanni Giobbe

University College London https://orcid.org/0000-0001-6958-7303

Yixiao Dong

Shanghai Institute for Advanced Immunochemical Studies (SIAIS), ShanghaiTech University

\section{Federica Michielin}

University College London

\section{Luca brandolino}

Univerity of Padova

\section{Michael Magnussen}

University College London

\section{Onelia Gagliano}

Univerity of Padova https://orcid.org/0000-0002-2571-3176

\section{Giulia Selmin}

Stem Cell and Regenerative Medicine Section, Great Ormond Street Institute of Child Health, University College of London, London WC1N 1EH, UK

\section{Valentina Scattolini}

University of Padova https://orcid.org/0000-0002-0855-9402

\section{Paolo Raffa}

University of Padova

\section{Soichi Shibuya}

UCL Great Hormond Street Institute of Child Health https://orcid.org/0000-0002-4274-1312

\section{Xuechun Wang}

Shanghai Institute for Advanced Immunochemical Studies (SIAIS), ShanghaiTech University Ju Qu

Shanghai Institute for Advanced Immunochemical Studies (SIAIS), ShanghaiTech University

\section{Marko Nikolic}

University College London https://orcid.org/0000-0001-6304-6848 


\section{Marco Montagner}

University of Padova https://orcid.org/0000-0003-3080-2138

Hans Clevers

Hubrecht Institute for Developmental Biology and Stem Cell Research https://orcid.org/0000-00023077-5582

Monica Giomo

University of Padova https://orcid.org/0000-0002-7690-1054

Paolo De Coppi

University College London https://orcid.org/0000-0002-1659-0207

Article

Keywords: hydrogel, tissue architecture, organoids, organ-like cultures

Posted Date: October 29th, 2021

DOI: https://doi.org/10.21203/rs.3.rs-952864/v1

License: (c) (1) This work is licensed under a Creative Commons Attribution 4.0 International License. Read Full License 
Four-dimensional hydrogel-in-hydrogel bioprinting for the spatiotemporal control of organoid and organotypic cultures

Anna Urciuolo ${ }^{1,2, \pi, *}$, Giovanni Giuseppe Giobbe ${ }^{3, \pi}$, Yixiao Dong ${ }^{4 \pi}$, Federica Michielin ${ }^{3}$, Luca Brandolino $^{5}$, Michael Magnussen ${ }^{3}$, Onelia Gagliano ${ }^{5}$, Giulia Selmin ${ }^{3}$, Valentina Scattolini ${ }^{2}$, Paolo Raffa $^{2}$, Soichi Shibuya ${ }^{3}$, Xuechun Wang ${ }^{4}, \mathrm{Ju} \mathrm{Qu}^{4}$, Marko Nikolic ${ }^{3}$, Marco Montagner ${ }^{1}$, Hans Clevers ${ }^{7}$, Monica Giomo ${ }^{5}$, Paolo De Coppi ${ }^{3,6}$, Nicola Elvassore ${ }^{3,4,5, *}$.

${ }^{1}$ Dept. of Molecular Medicine, University of Padova, Padova, Italy

${ }^{2}$ Istituto di Ricerca Pediatrica, Città della Speranza, Padova, Italy

${ }^{3} \mathrm{GOSICH}$ Zayed Centre for Research into Rare Disease in Children, University College London, London, UK

${ }^{4}$ Shanghai Institute for Advanced Immunochemical Studies (SIAIS), ShanghaiTech University, Shanghai, China

${ }^{5}$ Dept. of Industrial Engineering, University of Padova, Padova, Italy

${ }^{6}$ Dept. of Specialist Neonatal and Paediatric Surgery, Great Ormond Street Hospital, London, UK

${ }^{7}$ Hubrecht Institute, KNAW and University Medical Center, Utrecht, The Netherlands

I These authors contributed equally: Anna Urciuolo, Giovanni Giuseppe Giobbe \& Yixiao Dong

* Co-corresponding authors: Nicola Elvassore, n.elvassore@ucl.ac.uk;

Anna Urciuolo, anna.urciuolo@unipd.it 
Tissue architecture is a driving force for morphogenetic processes during development as well as for several physiological and regenerative responses. Far from being a passive static environment, tissue architecture is highly dynamic. Hydrogel technology reproduces in vitro geometrical and mechanical constrains that control the three-dimensional self-organization of (3D) organoids and organ-like cultures. This control is restricted to the initial culture conditions and cannot be adapted to the dynamic morphological changes of complex 3D cultures during their developmental trajectory. Here, we developed a method that overcomes this spatiotemporal limit. Using 2P crosslinking approach, high resolution 3D hydrogel structures can be fabricated within pre-existing hydrogel with spatiotemporal (fourdimensional, 4D) control relative to ex-vivo organotypic or organoid culture. This hydrogelin-hydrogel bioprinting approach enables to continuously instruct the self-organization of the evolving 3D organ-like cultures.

Three-dimensional (3D) organ-like cultures, including organoids and ex-vivo organotypic cultures, able to self-organize and recapitulate the structures and functionality of the tissue, providing physiologically relevant in vitro tissue models for the study of organ development, regeneration and diseases $^{1,2}$. The biological self-organization of organ-like cultures arises from progressive local interactions between cells in an initial environment. This is amplified by positive and negative feedbacks of biomechanical and biochemical factors, which steer the cellular self-organization in a highly context-dependent manner. Indeed, a dramatic influence on the final multicellular selforganization can be ascribed to molecular and environmental fluctuations within specific timewindows $^{3}$. In an apparent randomness of chaotic complex systems, such as in self-organizing organ-like cultures, the long-term prediction of cell culture behavior is uncertain. Recent efforts to overcome this limit have combined organogenesis knowledge and bioengineering-inspired culture systems ${ }^{4}$, with particular relevance for hydrogel-based approaches ${ }^{5}$. As an example, organoid experimental traceability, reproducibility and physiologic relevance has been recently improved by controlling the initial environment culture conditions via hydrogel-based technology ${ }^{6-8}$. However, the composition, mechanical properties and geometrical constrains of the engineered 3D scaffolds are pre-designed, and the existing approaches do not allow to precisely tailor hydrogel shape and properties according to the evolving development of the complex biological systems during cell culture.

Here, we developed a method to dynamically fabricate 3D hydrogel structures within hydrogel-based organ-like cultures. Newly fabricated hydrogels can be tailored to the specific culture system requests and at the desired culture time, matching the spatiotemporal requirement to control $3 \mathrm{D}$ organ-like cultures. With this technique we could fabricate natural or synthetic hydrogels that scale from sub-organoid (10 micrometer) to supra-organoid (millimeter) sizes with desired 3D shape and 
tunable mechanical properties able to guide and control cell behavior of 3D organ-like cultures. We named this technology hydrogel-in-hydrogel live 4D bioprinting.

We have previously shown how intravital 3D bioprinting can be applied in vitro for imposing initial 3D constrains on intestinal organoid growth ${ }^{9}$. We demonstrated that hydrogel fabrication can be achieved within Matrigel droplets (a gold standard hydrogel system for organoid culture) at the moment of cell seeding. Indeed, a photo-sensitive polymer, pre-mixed with Matrigel solution and upon Matrigel gelation at $37^{\circ} \mathrm{C}$, were cross-linked through two-photon (2P) mediated bioprinting 9 . We showed that, 7-hydroxycoumarin-3-carboxylic acid (HCCA) and 7-carboxymethoxy-4methylcoumarin (CMMC) conjugated with polymers, such as branched PEG and gelatin, respectively 7-Hydroxycoumarin-3-carboxylate-PEG (HCC-PEG) and HCC-Gelatin or CMMC-PEG and CMMCgelatin, can be efficiently crosslinked by $2 \mathrm{P}$ microscopy irradiation at the moment of cell seeding for organoid culture generation? ${ }^{9}$.

Starting from this proof of principle, we hypothesized that a photo-sensitive polymer could be loaded within the pre-existing solid hydrogel-based cell culture at the desired time point of the experiment to be crosslinked in 3D hydrogel structures. This is based on the idea that pre-existing solid hydrogel could allow free diffusion of soluble photo-sensitive polymers within their volume. Subsequently, photo-sensitive polymer crosslinking via femtosecond near-infrared tightly focused pulsed laser irradiation would enable the fabrication of 3D hydrogel-in-hydrogel structures (Fig. 1a). This strategy should allow the crosslinking of natural or synthetic hydrogels photosensitive polymer loaded into the 3D organ-like culture with complex geometry, additive manufacturing and tunable mechanical properties via 2P-microscope irradiation. Importantly, the temporal control of hydrogelin-hydrogel fabrication can be simultaneously combined with live imaging of the organ-like culture to define the positioning and orientation of the fabricating 3D hydrogel relative to a specific organoid, as well as to a defined portion of the organoid or to the ex-vivo organotypic culture. To assess the performance of this methodology on different culture conditions, we used organoids, including human liver, mouse intestinal and cancer organoids, as well as organotypic cultures of spinal cord and lung in Matrigel and collagen hydrogel. This provides an extensive validation of the proposed technology and address specific features of the experimental requests.

To test this hypothesis, we firstly investigated the self-diffusion coefficient of polymers in solid Matrigel droplets, which is the most used natural hydrogel used for 3D organoids culture. Fluorescence Recovery After Photobleaching (FRAP) analyses showed that fluorescein Isothiocyanate-Dextran (FITC-dextrans) with different molecular weight were freely diffusing within solid Matrigel according to the Fick's law, with a diffusion coefficient equal to $2.5 \pm 0.4 \mathrm{um} / \mathrm{s}^{2}$ or $1.4 \pm 0.1$ $\mathrm{um} / \mathrm{s}^{2}$ for 40 or $500 \mathrm{kDa}$ molecular weight FITC-dextrans respectively (Fig. 1b). These diffusivities, which are of the same order of magnitude of polymer in water, enable FITC-dextrans to reach the saturation in less than 300 seconds (5 minutes) within a Matrigel of $100 \mathrm{uL}$ droplets. 
Given this, we demonstrate that soluble photo-sensitive polymers were capable to diffuse within a pre-existing hydrogel and could be subsequently crosslinked in solid structures. We added liquid HCC-Gelatin on top of pre-existing hydrogel droplets (Matrigel) and incubated for 15 min before performing 3D printing. After incubation, HCC-Gelatin structures were fabricated within the Matrigel according to software design. The hydrogels showed a structural integrity over 2 days of incubation of the printed Matrigel droplet in DMEM as confirmed imaging analyses (Fig. 1c). The efficiency and the accuracy of 3D hydrogel-in-hydrogel photo-crosslinking was evaluated by fabricating hydrogel parallelepipeds of different heights and at multiple $Z$ positions (Fig. 1c). We also showed that hydrogels with minimal linewidth $(1,5 \pm 0,8 \mu \mathrm{m})$ can be fabricated as linear objects with scan or freeline scan modes (Fig. 1d). A resolution of $3 \mu \mathrm{m}$ between two scan lines was achieved (Fig. 1e). Accordingly, a 3D single line spiral-shaped hydrogel of HCC-Gelatin within Matrigel was fabricated using a computer drawing pad combined to a free line scan program of the multi-photon microscope (Fig. 1f). The reproducibility of hydrogel-in-hydrogel photo-crosslinking and of the fabricated structures was confirmed by the absence of swelling (Fig. 1g) and the preservation of the Young's modulus of HCC-Gelatin hydrogels printed within Matrigel just after (D0) or 2 days after Matrigel gelation (D2) (Fig. 1h). Following the same experimental strategy, we also confirmed that HCC-PEG and HCC-Gelatin hydrogels could undergo hydrogel-in-hydrogel printing in presence of living cells was confirmed with human hepatic organoid cultures $^{9-11}$ (Suppl. Fig. S1a). Figure $1 \mathrm{~h}$ and $1 \mathrm{l}$ shows that HCC-Gelatin loaded within the 3D organoid culture was crosslinked into the desired 3D shape and tailored on the selected organoids, preserving the overall organoid morphology (Suppl. Fig. S1b,c). Overall, these data demonstrated that hydrogel-in-hydrogel 3D bioprinting of photosensitive polymers can be efficiently achieved.

We first challenge the spatiotemporal complexity of hydrogel-in-hydrogel bioprinting in two 3D in vitro cell culture systems, which could require multiple interventions during the evolving culture: ex-vivo 3D organotypic spinal cord cultures and cancer cells in 3D in vitro systems. Ex-vivo 3D organotypic spinal cord cultures were first considered as a suitable model to investigate the possible effect of hydrogel-in-hydrogel fabrication in a dynamic event such as the 3D neural axon sprouting ${ }^{12}$. Such organotypic cultures retain the multicellular and structural complexity of the native tissue ${ }^{13,14}$, and can be maintained in 3D culture up to 14 days to study neural axon sprouting ${ }^{12}$. Spinal cord sections were derived from fetal rats, embedded in Matrigel droplet and cultured for 2 days, to when neural axons started sprouting from the central body of the organotypic section (Suppl. Fig. S2a). At this stage - i.e. 2 days of culture, hydrogel-in-hydrogel 4D live bioprinting was performed to fabricate HCC-Gelatin parallelepiped-shaped structures perpendicularly oriented in respect to the central body of the organotypic section (Suppl. Fig. S2a). Samples were cultured for further 2 days - i.e. 4 days of culture, and neural axon sprouting was evaluated by imaging analysis (Fig. 2a,b and Suppl. Fig. S2b). Interestingly, neural axons showed a patterned organization only where the HCC-Gelatin 
hydrogels were present and according with the hydrogel shape (Fig. 2a,b). Quantification of neural axon directionality ${ }^{12}$ shows that hydrogel-in-hydrogel 4D live bioprinted structures allowed neural axons alignment, when compared to randomly organized neural projections located within the 3D space of the untreated volume of the Matrigel droplet (Fig. 2b). Innervation plays a pivotal role as a driver of tissue and organ development as well as a means for their functional control and modulation ${ }^{15,16}$. During innervation neural axons are guided by molecular, mechanical, and physical cues to reach their targets ${ }^{17}$. The understanding of the neural axon guidance remains a challenge. A major limit in the study of neural axon guidance remains the characterization of the precise temporal regulation of guidance signals in the $3 \mathrm{D}$ environment ${ }^{18}$. Our experimental approach offers a unique possibility to precisely control the spouting of neural projections within the time and the space, opening new perspective for deeper characterization and understanding of key players regulating the neural axon guidance in complex and dynamic 3D in vitro neural models.

Subsequently, we challenged our approach to serve as an innovative method to study how dynamic changes in tissue architecture shape the behavior of cancer cells in 3D in vitro systems, such as tumor spheroids. Current engineered approaches cannot be adapted to varying topology and space surrounding cancer cells over time and, thus, paths and architecture are fixed since the beginning of the experiments. Here, we cultured A549 lung adenocarcinoma-derived organoids to evaluate the effect of dynamically imposed geometrical constrains on organoid growth and cancer cell migration (Fig. 2c-g). Non-cell adhesive CMMC-PEG hydrogels were used in combination with collagen-based $3 \mathrm{D}$ cultures, strengthening the idea that hydrogel-in-hydrogel 4D live bioprinting platform can be expanded to other biomaterials. As first, one day after organoid culture a series of 3D CMMC-PEG pillars distanced $10 \mu \mathrm{m}$ from each other where fabricated within pre-existing hydrogel to confine the selected organoid (Suppl. Fig. 3a). Live imaging analysis showed that during growth, cancer organoids first contacted the fabricated hydrogels and subsequently stared deforming the HCC-PEG pillars until cancer cells could cross the geometrical constrain given by the pillars (Fig. 2c and Suppl. Video 1). Actin staining analysis showed that fist cell projection and later entire cancer cells can migrate from the organoid across the pillars (Fig. 2d). During the different stages of metastatic dissemination, cancer cells adapt their invasive and migratory strategies to the different $3 \mathrm{D}$ environments encountered ${ }^{19-21}$. We therefore hypothesized that additive hydrogel-in-hydrogel bioprinting performed at multiple spatiotemporal points of the cancer organoid culture could be used to mimic the dynamic hurdles posed by the invaded tissues. To do so, we fabricated a first array of pillars distanced $40 \mu \mathrm{m}$ from each other to confine a specific cancer organoid (Fig. 2e and Suppl. Fig. 3b). As soon as the growing organoid contacted the pillars (day 6 after first bioprinting), an additive hydrogel-in-hydrogel bioprinting was performed to generate CMMC-PEG pillars distanced $15 \mu \mathrm{m}$ from each other that enclose the organoid together with the previously fabricated CMMChydrogels (Fig. 2e and Suppl. Fig. 3b). Different from the previous results, live imaging analysis showed that after contacting the first array of distanced bioprinted pillars, growing organoids modified 
their shape embracing fabricated CMMC-hydrogels (Fig. 2e,f). As soon as the cancer organoid reached the second array of closer pillars, migratory events could be observed, as showed by actin imaging analysis of cell protrusion across the imposed geometrical constrain (Fig. 2e,g).

Altogether this data demonstrates that tracks of different width and pattern can easily be generated in the proximity of tumor spheroids. This could be exploited, for example, to impose mechanical confinement on cells with low cell-cell cohesion and drive jamming ${ }^{22}$ or to create tracks with different topology in the proximity of collectively migrating cells.

We then challenged the possibility to use our hydrogel-in-hydrogel bioprinting technology to generate hydrogel structures with supra-organoid size able to guide organoid morphogenesis. To reach this aim, we took advantage of the LGR5-EGFP-DTR mouse small intestinal organoids ( $\mathrm{mSIOs})^{23}$ that allow to exactly locate stem cells during budding, in order to discriminate between the crypt compartment and the differentiated villus portion ${ }^{24,25}$. This reporter cell line would allow a direct evaluation of how the geometric constrain impinges organoid morphogenesis and mSIOs budding. First, experimental optimization showed that HCC-Gelatin and non-adhesive HCC-hydrogel (PEG based $)^{9}$ structures impose a constraint on growing cystic mSIOs inducing budding and, from day 2 to day 7 of culture, mSIOs LGR5 cells segregation (Suppl. Fig. S4).

Then, we designed a complex supra-organoid 3D structure to mimic primordial small intestine development ${ }^{26}$ characterized by 3 crypt-shaped structures per single $50 \mu \mathrm{m}$ z plane (height), with an anticlockwise rotation of $60^{\circ}$ and $30^{\circ}$ every subsequent $z$ plane (Fig. 3a and Suppl. Fig. S5a). Three-dimensional design was faithfully reproduced on the printed gelatin structures, as shown by autofluorescence and bright field images (Fig. 3b, Suppl. Fig. S5a and Suppl. Video S2). We then transferred such design to $3 \mathrm{D} \mathrm{mSIO}$ culture, enclosing a single $\mathrm{mSIO}$ within the internal lumen of the bioprinted hydrogel. We followed the evolution of $\mathrm{mSIO}$ morphogenesis inside the HCC structure over days (Fig. 3b and Suppl. Fig. S5b). Induced mSIO budding took place in correspondence of the 3D printed crypt shapes, with LGR5 cells leading to the protrusion and invasion of the overimposed HCC-Gelatin apertures. Therefore, mSIOs were able to adapt their shape to both circular central parts, and the branched sides of the primordial intestine-shaped hydrogels during the days of culture (Fig. 3b-e). Stability of the primordial intestine-shaped hydrogel was confirmed by the absence of statistically significant difference in swelling during the days of culture (Suppl. Fig. S5c). To test whether we could control the ability of $\mathrm{mSIO}$ budding in such structures, we quantified incremental size of the mSIOs within the available space of the primordial intestine-shaped hydrogels (Fig. 3c). From the quantification of the incremental central area occupied during the culture, we could assess that mSIOs were growing and adapting their shape to the central part of the hydrogels (Fig. 3d). We quantified in the same way the capacity of the mSIOs to invade the crypt-shaped portion of the primordial intestine-shaped hydrogel during culture (Suppl. Fig. S5d). We then evaluated the cell identity of the mSIOs after 10 days of culture. Staining for chromogranin 
A and lysozyme, markers of enteroendocrine and Paneth cells, respectively, confirmed that cellular identity was maintained (Suppl. Fig. S5e). Based on the previous results in which the constraints were guiding mSIOs budding, we further confirmed the specific localization of the differentiating intestinal cells by immunofluorescence. Importantly, we assessed specific expression of crypt stem cell markers olfactomedin 4 and LGR5-GFP only in the crypt-shaped portion of the primordial intestine hydrogel. Conversely, villic differentiation was restricted to the central region of the structure, as confirmed by the fatty acid-binding protein 1 staining (Fig. 3f). Increasing complexity of the printed structures allows fine control of the budding in small intestinal organoids. This feature strictly relates to in vivo curvature angles, where it is known that apical constriction and bending gradient induce budding formation in the intestine ${ }^{27,28}$. Our technology could allow to design structures that permit the study of the curvatures that instruct small intestinal organoid budding.

Based on the ability of geometrical constraints to control organ and organoid morphogenesis and biological cell responces ${ }^{29}$, we investigated if hydrogel-in-hydrogel live bioprinting could be used to control hepatocyte polarization ${ }^{30}$ in human fetal hepatocyte organoid 3D cultures. Mechanically disaggregated hepatocyte organoids were seeded in Matrigel, and live bioprinting was performed to generate HCC-Gelatin hydrogels as distant walls or adjacent pillar structures around the forming organoids (Fig. 4a,b). The samples were kept in expansion medium for 6 days and differentiated for a further $24 \mathrm{~h}$. Imaging analysis showed preservation of hydrogel integrity and human liver organoid morphology after bioprinting (Fig. 4a,b). Organoids grew and remodeled their shape according to the constrains imposed by the bioprinted structures (Fig. 4a). Interestingly, in presence of HCCGelatin hydrogels but in absence of constrain - i.e. distant walls, no apical-basal polarization of multidrug resistance-associated protein 2 (MRP2) and zonula occludens-1 (ZO-1) was observed. Conversely, we observed polarized cells in correspondence of the interacting HCC-gelatin pillars, with expression of MRP2, ZO-1 and f-actin (ACT) towards the internal portion of the organoid, while $\beta 4$-integrin (INTß4) remained confined on the basal layer (Fig. 4b). Some polarized behavior was observed when the walls were printed adjacent to the growing organoid (Suppl. Fig. S6). Cell polarity requires proper compartmentalization of basolateral and apical proteins, as well as junction formation ${ }^{31}$. Recapitulation of correct polarization within organoids is essential to mimic organ function (i.e. mucus, acid, hormone, or bile acid secretion, depending on the tissue) in order to build a reliable and functional in vitro model.

Then, we investigated the possible biological effect of live bioprinting on ex vivo organotypic cultures of mesenchyme-free lung epithelium rudiments and human fetal hepatocyte organoids. We coupled the 3D organotypic culture of lung epithelium rudiments in Matrigel drops with the photo-printing of 8-arm HCC-PEG structures to control the direction of spontaneous branching under previously optimized culture conditions ${ }^{32}$. Specifically, circular pillars were printed in the close proximity of bud 
tips and branching was monitored during the first cycle of epithelial budding (Fig. 4c). Time-lapse imaging over 3 days with five-hours intervals shows the controlled direction of branching as a consequence of the physical interaction with the pillar during bud tip bifurcation (Fig. $\mathbf{4 d}$ and Suppl. Video S3). Induced branching allows the preservation of the cytoskeletal apical polarity upon interaction with the pillars (Fig. 4e). Moreover, expression of the bud-tip cell marker Sox9 was downregulated in correspondence of the bifurcation point induced by the pillars during branching progression, with increasing Sox9 downregulation according to the cleft depth (Fig. 4f). Overall, these data showed that hydrogel-in-hydrogel bioprinting allowed the fabrication of 3D structures with a sub-organ-like sized and with desired shape and orientation able to guide cell behavior in organotypic and organoid cultures. Lung branching morphogenesis shapes the respiratory tree through the reiterative formation of branches from the surface of an epithelial bud tip and the subsequent branch outgrowth into the surrounding mesenchyme, according to a remarkably stereotyped and genetically hardwired process $^{33}$. Here, we showed that branching morphogenesis can be guided ex vivo by forcing the bifurcation of bud tips through 3D-printed circular pillars within. We confirmed the preservation of the cytoskeletal apical polarity, a prerequisite for branching initiation ${ }^{34}$, as well as the loss of the bud tip identity represented by Sox 9 downregulation ${ }^{35}$ in tip cells undergoing bifurcation through the pillars. Our 3D model of guided branching provides a new tool to investigate extracellular regulators of lung branching morphogenesis, such as interactions with extracellular matrix (EMC) components and physical forces ${ }^{36-38}$, that are increasingly recognized to integrated into genetic patterns governing branching, yet poorly understood.

Here, we showed a method to fabricate hydrogel-in-hydrogel using photosensitive polymers that diffuse within pre-existing hydrogels and that crosslink via near-infrared multiphoton absorption in 3D structures thanks to the use of a multiphoton microscope. This experimental setup performed with different $2 \mathrm{P}$ microscopes and biomaterials allows simultaneously to $i$ ) perform live imaging of the organ-like 3D culture, ii) define the positioning and orientation of the desired hydrogel relative to a specific organoid with sub- or supra-organoid resolution, and tunable mechanical properties, iii) perform single or additive hydrogel-in-hydrogel bioprinting at the desired time point of the organ-like 3D culture. 


\section{Materials and methods}

Hydrogel Preparation. HCC-Gelatin, HCC-PEG and CMMC-PEG were synthesized as previously described (Urciuolo et al., 2020). Briefly, 7-hydroxycoumarin-3-carboxylic acid (HCCA) was conjugated to gelatin from porcine skin. HCCA and 7-carboxymethoxy-4-methylcoumarin (CMMC) were conjugated to $10 \mathrm{kDa}$ PEG amine tripentaerythritol core (4-arm PEG-NH${ }_{2}$ ) or 20kDA 8-arm PEG amine tripentaerythritol core (8arm-PEG- $\mathrm{NH}_{2}$ ) to create biocompatible photosensitive compounds with alternate organic conjugated components (HCC-Gel, HCC-4-arm PEG, HCC-8-arm PEG, CMMC-4-arm PEG, CMMC-8-arm PEG) that polymerises at defined wavelengths. After synthesis, freeze dried powdered stock were combined with PBS at concentration of $10 \% \mathrm{w} / \mathrm{v}$ for HCC-Gelatin and 30\% for HCC-4-arm PEG or HCC-8-arm PEG or CMMC-4-arm PEG or CMMC-8-arm PEG. The resulting solutions were agitated at $800 \mathrm{rpm}$ at $37^{\circ} \mathrm{C}$ for PEG-based polymers or at $60^{\circ} \mathrm{C}$ for Gelatinbased polymers until dissolving. The final solutions were kept at $4^{\circ} \mathrm{C}$, protected from light. HCCGelatin was reheated at $60^{\circ} \mathrm{C}$ for 10 minutes before use.

Fluorescence Recovery After Photobleaching (FRAP) analysis. A drop of $3 \mu$ of Matrigel (Corning) was cast on a glass coverslip (Bio Optica) equipped with custom-made polydimethylsiloxane well ( $3.5 \mathrm{~mm}$ diameter, $6 \mathrm{~mm}$ hight) and incubated at $37^{\circ} \mathrm{C}$ for 4 minutes to allow MRF gelation. Subsequently, $15 \mu$ s solution of either fluorescein isothiocyanate (FITC)-dextran $40 \mathrm{kDa}$ (2mg/ml in PBS, Sigma) or FITC-dextran $500 \mathrm{kDa}$ (2mg/ml in PBS, Sigma) were loaded over the Matrigel droplet located within the PDMS well and incubated at $37^{\circ} \mathrm{C}$ for 15 minutes.

For FRAP experiments we employed a Leica SP5 confocal microscope, equipped with an Argon laser $488 \mathrm{~nm}, 30 \mathrm{~mW}$ nominal power. The experiments were carried out with a 10x AIR objective, with $600 \mathrm{~Hz}$ scanning frequency at $90 \%$ of laser power (13\% used for pre- and post-bleaching acquisition, $100 \%$ used for bleaching), and 10 frames of pre-bleaching acquisition, 20 frames of bleaching and 100 frames of post-bleaching acquisition (time between frames was set as 3 seconds). The bleaching areas was defined as follow: $30 \mu \mathrm{m}$ in diameter circle, bleached with "Zoom-in mode", $100 \mu \mathrm{m}$ bleaching z-plane. Fluorescence recovery was measured at the center of the bleached area consisting of a round ROI $30 \mu \mathrm{m}$ in diameter. FRAP quantification is shown as the result of 3 independent experiments, with 5 measurement per each Matrigel droplet.

Atomic force measurement. A liquid drop of HCC-Gelatin was added onto freshly prepared $100 \%$ Matrigel solid drops or onto $100 \%$ Matrigel drops prepared 2 days before treatment and maintained in DMEM at $37{ }^{\circ} \mathrm{C}$. Treated samples were incubated at $37^{\circ} \mathrm{C}$ for 15 minutes. The volume ratio between photosensitive hydrogels and solid Matrigel drops was 2:1. Hydrogels were fabricate within the pre-existing Matrigel with drops by using Scientifica 2-Photon microscope according to our 
previous study ${ }^{9}$. For AFM measurements, Matrigel was depolymerized by using cold PBS-EDTA (0.5mM EDTA, Invitrogen \#AM9260G) under gentle agitation overnight at $4^{\circ} \mathrm{C}$ as previously shown ${ }^{9}$. All the samples were analysed by using an Atomic Force Microscope, mounted on an Inverted Optical Microscope (XEBio, Park Systems, Korea) as previously reported ${ }^{9}$.

\section{Organ-like cultures}

Intestinal and liver organoid cultures. For mouse small intestinal organoids, LGR5-DTR-EGFP mice $^{24}$ were sacrificed by cervical dislocation and the intestine was harvested from the pylorus to the caecum, as previously reported ${ }^{25}$. The tissue was washed in ice-cold PBS (Thermo Fisher), cleared of any mesenteric or fatty tissue and cut-open longitudinally. A glass coverslip was used to remove the villi from the luminal mucosa and thoroughly washed in PBS. Tissue was further cut into 2-3 mm pieces and incubated in $2 \mathrm{mM}$ ethylenediaminetetraacetic acid (EDTA - Sigma) in PBS for $30 \mathrm{~min}$ at $4^{\circ} \mathrm{C}$. EDTA was removed and tissue was vigorously shacked for $5 \mathrm{~min}$ in PBS with calciummagnesium (Thermo Fisher). The supernatant containing the crypts was collected and centrifuged at $800 \mathrm{rpm}$ for $5 \mathrm{~min}$ at $4^{\circ} \mathrm{C}$ (Hettich zentrifugen Rotina 420). The pellet was washed once with basal media Advanced DMEM/F12 media, supplemented with $1 \%$ of GlutaMAX, HEPES and Penicillin/Streptomycin (ADMEM +/+/+) and centrifuged at $1000 \mathrm{rpm}$. The pellet was re-suspended

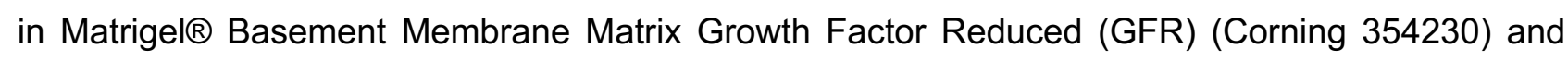
plated onto a 24-well plate. Primocin $1 \mathrm{X}$ (Thermo Fisher) and ROCK inhibitor $10 \mu \mathrm{m}$ were added after isolation. All the reagents supplemented for the mouse small intestinal organoid cultures are reported in Supplementary Table S1. For human fetal hepatocyte organoids, human fetal livers were collected via the Joint MRC/Wellcome Trust Human Developmental Biology Resource under informed ethical consent with Research Tissue Bank ethical approval (18/LO/0822, project 200525). 8 post conception week (PCW) liver was washed in PBS, cut into 1-2 mm cubes and digested for 10 minutes in HBSS (Thermo Fisher) + EDTA $2 \mathrm{mM}$ at $37^{\circ} \mathrm{C}$. HBSS was discarded and liver cubes were further dissociated in collagenase type 1 (Sigma) for $10 \mathrm{~min}$ at $37^{\circ} \mathrm{C}$. Liver cubes were pipetted thoroughly to facilitate dissociation until a single cell solution was obtained. Cells were pelleted at $800 \mathrm{rpm}$ for $5 \mathrm{~min}$ at $4^{\circ} \mathrm{C}$ and washed twice in ADMEM $+/+/+$ ). Finally, cell pellet was resuspended in Matrigel and plated in 24-well plates. Primocin 1X (Thermo Fisher) and ROCK inhibitor $10 \mu \mathrm{m}$ were added after isolation. Liver organoids were cultured following the protocol previously published ${ }^{10}$. Cells were passaged every 6-8 days. All the reagents supplemented for the human liver organoid cultures are reported in Supplementary Table S2. To passage the organoids, Matrigel droplets were thoroughly disrupted by pipetting in the well and transferred to tubes in ice. Cells were washed with $10 \mathrm{~mL}$ of ice-cold ADMEM $+/+/+$ and spin at $200 \mathrm{~g}$ at $4^{\circ} \mathrm{C}$. For single cell disaggregation, organoid pellets were incubated in TrypLE Express (Thermo Fisher) for 5 min at $37^{\circ} \mathrm{C}$. Organoids were thoroughly pipetted to help disaggregation, then single cells were spin at $200 \mathrm{~g}$ at $4^{\circ} \mathrm{C}$ and plated in Matrigel droplets. Following single cell dissociation, ROCK inhibitor $10 \mu \mathrm{m}$ was added to 
the culture medium. For mechanical disaggregation into cell clumps, the pellet was resuspended in $1 \mathrm{~mL}$ of cold ADMEM +/+/+ and organoids were manually disrupted by narrow (flamed) glass pipette pre-wet in BSA $1 \%$ in PBS, to avoid adhesion to the glass. Cells were washed, pelleted and supernatant was discarded. Almost-dry pellets of disaggregated organoids were thoroughly resuspended in cold liquid Matrigel, aliquot in 30-40 $\mu \mathrm{L}$ droplets in Petri dishes, and incubated at $37^{\circ} \mathrm{C}$ for $30 \mathrm{~min}$ to form a gel.

Cancer organoid cultures. A549 lung adenocarcinoma cells (ATCC) were cultured in RPMI 1640 medium supplemented with $10 \%$ of fetal bovine serum (FBS) and $1 \%$ of Penicillin/Streptomycin (P/S). For organoids generation, mycoplasma-free A549 cells were seeded in $90 \%$ Matrigel (Corning $\AA$ ) at a final concentration of $0.3 \times 10^{6}$ cells $\mathrm{mL}^{-1}$. The cell-embedded Matrigels $(25 \mu \mathrm{L}$ each) were transferred into a 24-well culture plate and incubated at $37{ }^{\circ} \mathrm{C}$ in $5 \% \mathrm{CO}_{2}$ with medium change every 2-3 days. After 7 days, Matrigels were dissolved by using Cell Recovery Solution (Corning ${ }^{\circledR}$ ) for $20 \mathrm{~min}$ on ice. The organoids were gently resuspended in culture medium, and reembedded in collagen hydrogel at a density around 40 organoids per hydrogel. To prepare the collagen hydrogel, organoids were mixed with type I collagen (rat tail derived, Thermos Fisher) solution and adjusted to neutral $\mathrm{pH}$ by $\mathrm{NaOH}(1 \mathrm{~N})$ to form a physical cross-linked hydrogel with a final concentration of $2.4 \%(\mathrm{w} / \mathrm{v})$ after incubating at $37^{\circ} \mathrm{C}$ for $20 \mathrm{~min}$. The hydrogels (12 $\mu \mathrm{L}$ each) were cultured with culture medium at least $24 \mathrm{~h}$ before printing.

Mouse fetal lung organotypic cultures. All animal experiments were performed by personnel having UK Home Office Personal Licence (PIL I7ED92582) in line with ethical approval. Wild type CD-1 mice were mated and marked as E0.5 pregnant when they presented a vaginal plug. Pregnant mice were euthanized by cervical dislocation at E12.5, corresponding to pseudoglandular stage of lung development, and lung epithelium rudiments were cultured as previously reported ${ }^{32}$. Briefly, embryos were extracted and lungs harvested under a stereomicroscope. Lungs were washed in PBS and subsequently treated with $8 \mathrm{U} / \mathrm{mL}$ Dispase (ThermoFisher Scientific) for 2 min at room temperature. Mesenchymal tissue was removed with tungsten needles (Interfocus) and lung epithelium rudiments were isolated and embedded in a $5 \mu \mathrm{L}$ MRF on sterile coverslips. After MRF gelation, 250 $\mu \mathrm{L}$ of DMEM/F12 supplemented with $1 \% \mathrm{p} / \mathrm{s}, \quad 0.1 \%$ BSA, $1 \%$ Insulin-Transferrin-Selenium (ITS) (ThermoFisher Scientific), 200ng/mL human Recombinant FGF10 (Peprotech) and $1 \mu \mathrm{M}$ CHIR99021(Tocris). All the reagents supplemented for the mouse fetal lung organotypic cultures are reported in Supplementary Table S3.

Rat fetal spinal cord organotypic cultures. Rat fetal spinal cords were isolated and culture as previously described ${ }^{12}$. Briefly, isolated spinal cords were cut in three sections and cultured within $15 \mu \mathrm{L}$ drop of $100 \%$ Matrigel Growth Factor Reduced (Corning 354 230) casted onto glass coverslips. Samples were cultured in Neurobasal medium (Gibco 21103 049), B-27 supplement (Gibco 17504 044) 1X, 2\% Horse serum (Gibco 16050 122), 0.5 mM Glu- taMAX Supplement

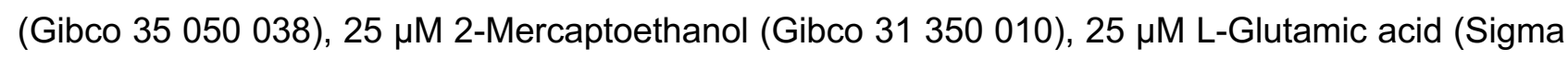


G5889), Gentami- cin/Amphotericin (Gibco R01510), $10 \mathrm{ng} / \mathrm{mL}$ ciliary neurotrophic fac- tor (CNTF, PeproTech 450-13), and $10 \mathrm{ng} / \mathrm{mL}$ glial-cell-line-derived neurotrophic factor (GDNF, Peprotech 45010).

Hydrogel-in-hydrogel bioprinting. Organoid or organotypic culture pre-seeded into droplets of hydrogel (Matrigel or collagen) were cultured onto $13 \mathrm{~mm}$ coverslips in 24-well plates. On the day of the print, coverslips were removed with sterile tweezers, dried with sterile paper and incubated with photosensitive-polymers. For photosensitive hydrogel diffusion, a liquid drop of HCC-PEG or HCCGelatin was added onto the 3D culture and incubated at $37^{\circ} \mathrm{C}$ for 15 minutes (Matrigel-based cell cultures) or 4 hours (Collagen-based cell cultures). The volume ratio between photosensitive hydrogels and solid hydrogel drops was 2:1. The multiphoton microscopes used for 2P-mediated hydrogel crosslinking were Scientifica 2-Photon microscope, Zeiss.Examiner.Z1 Multiphoton LSM880 Confocal equipped with Solent Scientific Incubator Chamber and Multiphoton Leica SP8 confocal microscope. After the bioprinting, the coverslip was moved into another $6 \mathrm{~mm}$ petri dish filled with $10 \mathrm{ml}$ of basal media and incubated at $37{ }^{\circ} \mathrm{C}$ for 5 minutes to remove the un-polymerized photosensitive-polymer. Finally, the coverslip was put in a 24 multi-well plate with $500 \mu$ l of organoid or organotypic culture media.

Multiphoton Polymerising Settings. For experiments performed with murine small intestine and human fetal liver organoids, and murine lung organotypic cultures, we used Zeiss.Examiner.Z1 Multiphoton LSM880 Confocal. Optimized settings for polymerization in murine small intestine and human fetal liver organoid samples were as follows: pixel resolution 1640x1640, pixel dwell time $0.98 \mu \mathrm{s}$, Zoom 1.2X, laser wavelength $800 \mathrm{~nm}$, stack depth $2.57 \mu \mathrm{m}$, laser power $67 \%$ for Gelatinbased hydrogels and $40 \%$ for PEG-based hydrogels, 10X immersion objective. Optimized settings for polymerization murine lung organotypic culture samples were as follows: pixel resolution

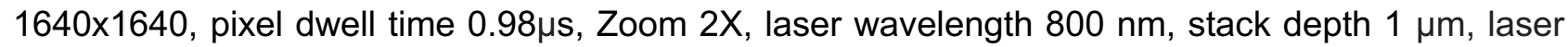
power $40 \%$ for PEG-based hydrogels, 4X immersion objective. Hydrogels were crosslinked by imaging designed ROls generated by the Zeiss software. Z-stack depth was specifically defined on the basis of 2P-live imaging and according to the organoid size. For experiments performed with cancer organoids, we used the Multiphoton Leica SP8 confocal microscope. Pillar structures were printed around the organoids according with the design by using the multiple ROls generated by the Leica software. For experiments performed with spinal cord cultures, Scientifica 2-Photon microscope. Hydrogels were fabricated according to our previous study ${ }^{9}$.

Immunofluorescence and imaging acquisition. Matrigel droplets with embedded photo-printed organoids were fixed in $0.5 \%$ glutaraldehyde dissolved in PBS with $\mathrm{Ca}^{++} / \mathrm{Mg}^{++}$for $15 \mathrm{~min}$ at $\mathrm{RT}$, and 
then extensively washed in PBS. Free aldehydes were quenched with an incubation of $0.1 \mathrm{M} \mathrm{NH}_{4} \mathrm{Cl}$ for 1 hour. Whole mount staining was performed by blocking and permeabilizing the cells with PBSTriton $0.5 \%$ with BSA $1 \%$ for $2 \mathrm{~h}$ at room temperature (RT). Primary antibodies were incubated in blocking buffer for $48 \mathrm{~h}$ at $4{ }^{\circ} \mathrm{C}$ in rotation, and then extensively washed in PBS-Triton $0.5 \%$ at RT. Secondary antibodies were incubated overnight at $4^{\circ} \mathrm{C}$ in rotation and extensively washed. The list of the used antibodies is reported in Supplementary Table S4. Samples were mounted in TDX mounting medium (Sigma Aldrich). Bright field images and EGFP fluorescence images were acquired at a Leica DMIL microscope and DFC420C camera. Immunofluorescence and live imaging were acquired by using Zeiss LSM 710, Zeiss.Examiner.Z1 Multiphoton LSM880 Confocal or Multiphoton Leica SP8 confocal microscopes.

Imaging preparation and analysis. We used ImageJ software for adjustments of levels and contrast, maximum and standard deviation intensity projections and 3D reconstructions. All the quantification reported have been performed with ImageJ software. Quantification of hydrogel swelling was performed by measuring the area of the hydrogels. For directionality analysis, Directionality Image J plugin was used to analyse bright field and TUJ-1 immunofluorescence images of the neural projections departing from the central body of the spinal cord within the volume the Matrigel, in presence or absences of HCC-Gelatin hydrogels. The measurement were performed as previously described ${ }^{12}$. Images with completely isotropic content are expected to give a flat histogram, whereas images in which there is a preferred orientation are expected to give a histogram with a peak at that orientation. The quantification was expressed as the mean of 4 to 5 independent biological replicates.

Statistical analysis. All analyses were performed with GraphPad prism 6. We expressed data as mean \pm s.e.m or mean \pm s.d of multiple biological replicates (as indicated in the figure legends). We determined statistical significance by unequal variance Student's t-test, one-way analysis of variance (ANOVA) and Tukey's multiple comparison test or two-way ANOVA and Sidak's multiple comparisons test. $P$ value lower than 0.05 was considered statistically significant.

\section{Data availability}

The main data supporting the results in this study are available within the paper and its Supplementary Information. The raw image data and the analysed data generated in this study are available from the corresponding author upon reasonable request.

\section{Acknowledgements}


This work was supported by 2017 STARS-WiC grant of University of Padova, Progetti di Eccellenza CaRiPaRo, TWINING of University of Padova, Oak Foundation Award (grant no. W1095/OCAY-14191), 'Consorzio per la Ricerca Sanitaria' (CORIS) of the Veneto Region, Italy (LifeLab Program) to N.E. and the STARS Starting Grant 2017 of University of Padova (grant code LS3-19613) and IRP Consolidator Grant (grant code: 21/05 Irp) to AU. We thank Prof. Frederic de Sauvage for providing Lgr5-DTR-GFP mice. GGG was supported by the NIHR Great Ormond Street Hospital Biomedical Research Centre and the BRC Catalyst Fellowship. PDC and NE were supported by the Oak award W1095/OCAY-14- 191. PDC is supported by NIHR Professorship and the GOSH Children's Charity. F.M. is supported by a NIHR BRC Catalyst Fellowship and a Rosetrees Trust grant (grant code Seedcorn2020/100052). S.S is supported by Japan society for the promotion of science overseas research fellowships (310072). All research at Great Ormond Street Hospital NHS Foundation Trust and University College London GOSICH, Zayed Centre for Research into Rare Disease in Children is made possible by the NIHR Great Ormond Street Hospital Biomedical Research Centre. The views expressed are those of the author(s) and not necessarily those of the National Health Service, the NIHR or the Department of Health. We would like to thank the Human Developmental Biology Resource (HDBR).

\section{Author contributions}

AU, GGG, XY and NE designed the study. GG derived and characterized liver and intestine organoid cultures. $\mathrm{LB}$ and PR contributed to $4 \mathrm{D}$ printing characterization experiments. $\mathrm{OG}, \mathrm{M}$ Magnussen and GS contributed to the organoid printing experiments. FM design the experiment with fetal lung epithelium. FM and SS performed the experiments with the fetal lung epithelium. AU, PR and VS performed the experiments with organotypic spinal cord cultures. $\mathrm{XY}, \mathrm{XW}$ and JQ performed the experiments with cancer organoids. AU, GGG and NE wrote the manuscript. M Montagner, HC, MN, PDC helped to design the experiments and critically discussed the manuscript. $A U$ and NE supervised the project.

\section{Competing interests}

NE and OG have an equity stake in ONYEL Biotech s.r.I. AU and NE submitted a patent for the intravital 3D bioprinting (provisional patent number 102020000008779). 


\section{Bibliography}

1. Rossi, G., Manfrin, A. \& Lutolf, M. P. Progress and potential in organoid research. Nat. Rev. Genet. 19, 671-687 (2018).

2. Shamir, E. R. \& Ewald, A. J. Three-dimensional organotypic culture: Experimental models of mammalian biology and disease. Nat. Rev. Mol. Cell Biol. 15, 647-664 (2014).

3. Brassard, J. A. \& Lutolf, M. P. Engineering Stem Cell Self-organization to Build Better Organoids. Cell Stem Cell 24, 860-876 (2019).

4. Moysidou, C. M., Barberio, C. \& Owens, R. M. Advances in Engineering Human Tissue Models. Front. Bioeng. Biotechnol. 8, (2021).

5. Park, Y., Huh, K. M. \& Kang, S. W. Applications of biomaterials in 3d cell culture and contributions of $3 \mathrm{~d}$ cell culture to drug development and basic biomedical research. Int. J. Mol. Sci. 22, 1-21 (2021).

6. Nikolaev, M. et al. Homeostatic mini-intestines through scaffold-guided organoid morphogenesis. Nature 585, 574-578 (2020).

7. Brassard, J. A., Nikolaev, M., Hübscher, T., Hofer, M. \& Lutolf, M. P. Recapitulating macroscale tissue self-organization through organoid bioprinting. Nat. Mater. 1-8 (2020). doi:10.1038/s41563-020-00803-5

8. Brandenberg, N. et al. High-throughput automated organoid culture via stem-cell aggregation in microcavity arrays. Nat. Biomed. Eng. 4, 863-874 (2020).

9. Urciuolo, A. et al. Intravital three-dimensional bioprinting. Nat. Biomed. Eng. (2020). doi:10.1038/s41551-020-0568-z

10. Hu, H. et al. Long-Term Expansion of Functional Mouse and Human Hepatocytes as 3D Organoids. Cell 175, 1591-1606.e19 (2018).

11. Hendriks, D., Artegiani, B., Hu, H., Chuva de Sousa Lopes, S. \& Clevers, H. Establishment of human fetal hepatocyte organoids and CRISPR-Cas9-based gene knockin and knockout in organoid cultures from human liver. Nat. Protoc. (2020). doi:10.1038/s41596-020-00411-2

12. Raffa, P. et al. Decellularized skeletal muscles display neurotrophic effects in threedimensional organotypic cultures. Stem Cells Transl. Med. 1-11 (2020). doi:10.1002/sctm.20-0090

13. Al-Ali, H., Beckerman, S. R., Bixby, J. L. \& Lemmon, V. P. In vitro models of axon regeneration. Exp. Neurol. 287, 423-434 (2017).

14. Pandamooz, S., Nabiuni, M., Miyan, J., Ahmadiani, A. \& Dargahi, L. Organotypic Spinal Cord Culture: a Proper Platform for the Functional Screening. Mol. Neurobiol. 53, 46594674 (2016).

15. Doblado, L. R., Martínez-Ramos, C. \& Pradas, M. M. Biomaterials for Neural Tissue 
Engineering. Front. Nanotechnol. 3, (2021).

16. Das, S. et al. Innervation: the missing link for biofabricated tissues and organs. npj Regen. Med. 5, (2020).

17. Bonanomi, D. Axon pathfinding for locomotion. Semin. Cell Dev. Biol. 85, 26-35 (2019).

18. Stoeckli, E. T. Understanding axon guidance: Are we nearly there yet? Dev. 145, (2018).

19. Drost, J. \& Clevers, H. Organoids in cancer research. Nat. Rev. Cancer 18, 407-418 (2018).

20. Lintz, M., Muñoz, A. \& Reinhart-King, C. A. The Mechanics of Single Cell and Collective Migration of Tumor Cells. J. Biomech. Eng. 139, 1-9 (2017).

21. Mosier, J. A., Schwager, S. C., Boyajian, D. A. \& Reinhart-King, C. A. Cancer cell metabolic plasticity in migration and metastasis. Clin. Exp. Metastasis 38, 343-359 (2021).

22. Ilina, O. et al. Cell-cell adhesion and 3D matrix confinement determine jamming transitions in breast cancer invasion. Nat. Cell Biol. 22, 1103-1115 (2020).

23. Sato, T. et al. Single Lgr5 stem cells build crypt-villus structures in vitro without a mesenchymal niche. Nature 459, 262-265 (2009).

24. Tian, H. et al. A reserve stem cell population in small intestine renders Lgr5-positive cells dispensable. (2012). doi:10.1038/nature10408

25. Giobbe, G. G. et al. Extracellular matrix hydrogel derived from decellularized tissues enables endodermal organoid culture. Nat. Commun. 10, 5658 (2019).

26. Shyer, A. E. et al. Villification: How the Gut Gets Its Villi. Science (80-. ). 342, 212-218 (2013).

27. Hartl, L., Huelsz-Prince, G., van Zon, J. \& Tans, S. J. Apical constriction is necessary for crypt formation in small intestinal organoids. Dev. Biol. 450, 76-81 (2019).

28. Shyer, A. E., Huycke, T. R., Mahadevan, L. \& Tabin Correspondence, C. J. Bending Gradients: How the Intestinal Stem Cell Gets Its Home. Cell 161, 569-580 (2015).

29. Chan, C. J., Heisenberg, C. P. \& Hiiragi, T. Coordination of Morphogenesis and Cell-Fate Specification in Development. Curr. Biol. 27, R1024-R1035 (2017).

30. Zeigerer, A. et al. Functional properties of hepatocytes in vitro are correlated with cell polarity maintenance. Exp. Cell Res. 350, 242-252 (2017).

31. Co, J. Y. et al. Controlling Epithelial Polarity: A Human Enteroid Model for Host-Pathogen Interactions. Cell Rep. 26, 2509-2520.e4 (2019).

32. Shibuya, S., Allen-Hyttinen, J., De Coppi, P. \& Michielin, F. In vitro models of fetal lung development to enhance research into congenital lung diseases. Pediatr. Surg. Int. 37, (2021).

33. Metzger, R. J., Klein, O. D., Martin, G. R. \& Krasnow, M. A. The branching programme of mouse lung development. Nature 453, (2008). 
34. Fumoto, K., Takigawa-Imamura, H., Sumiyama, K., Kaneiwa, T. \& Kikuchi, A. Modulation of apical constriction by Wnt signaling is required for lung epithelial shape transition. Dev. 144, (2017).

35. Rawlins, E. L., Clark, C. P., Xue, Y. \& Hogan, B. L. M. The Id2+ distal tip lung epithelium contains individual multipotent embryonic progenitor cells. Development 136, (2009).

36. Yamada, K. M. et al. Extracellular matrix dynamics in cell migration, invasion and tissue morphogenesis. International Journal of Experimental Pathology 100, (2019).

37. Wang, S., Sekiguchi, R., Daley, W. P. \& Yamada, K. M. Patterned cell and matrix dynamics in branching morphogenesis. The Journal of cell biology 216, (2017).

38. Nelson, C. M. et al. Microfluidic chest cavities reveal that transmural pressure controls the rate of lung development. Dev. 144, (2017). 
a
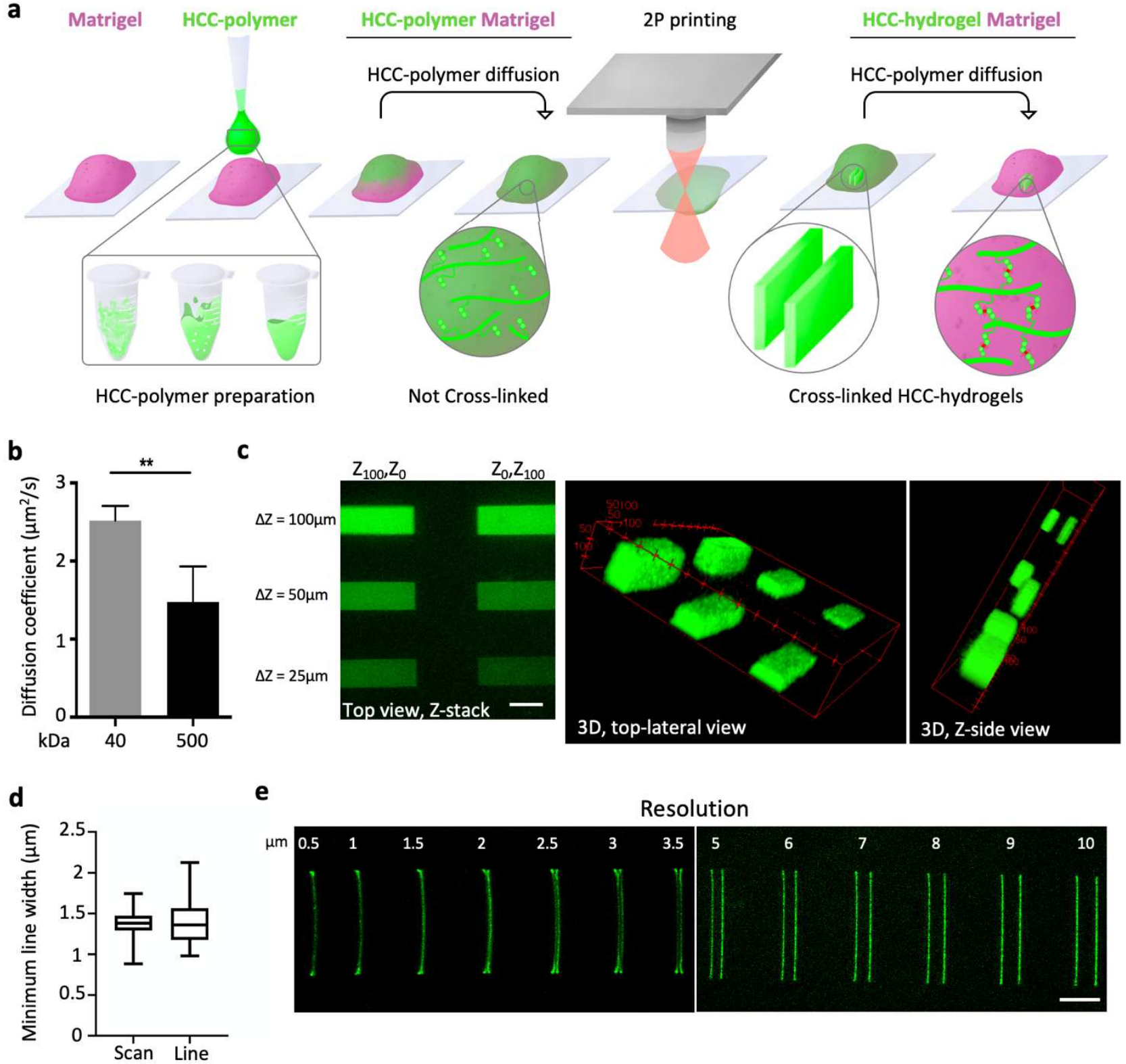

e

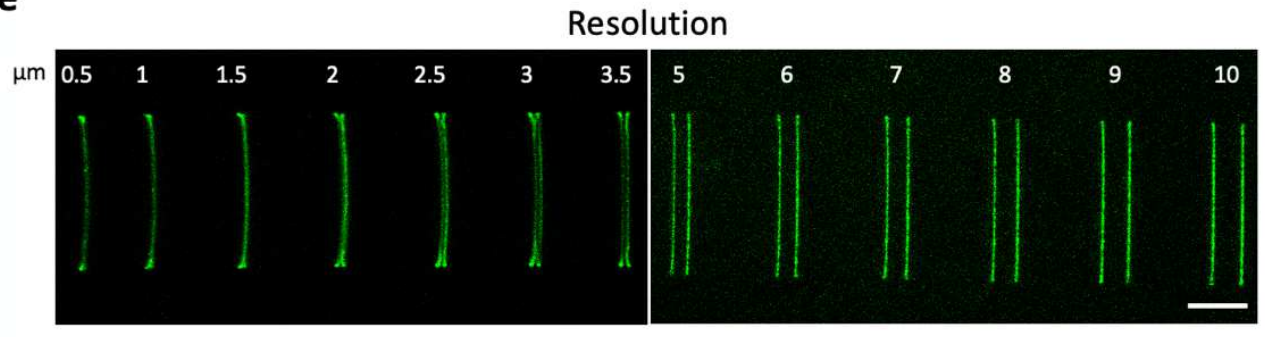

f

Freeline scanning

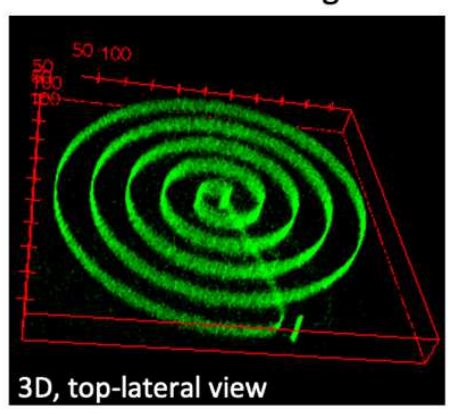

g

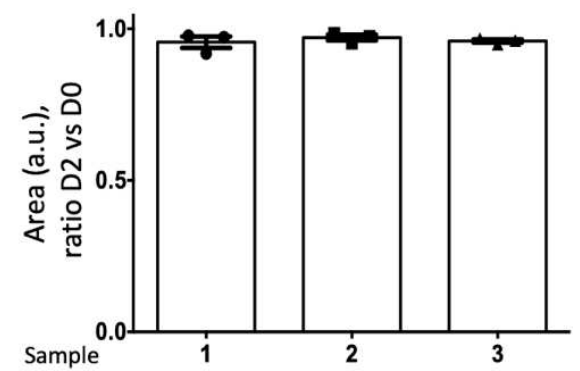

h

Stiffness during the time

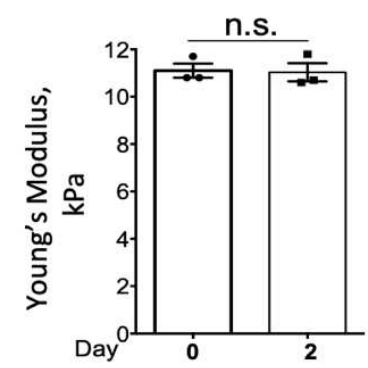

Figure 1.

Performance of hydrogel-in-hydrogel bioprinting in a 4D-fashion and integrated in organoid 3D cultures. a) Strategy and set-up for hydrogel-in-hydrogel live 4D bioprinting. HCC-hydrogel 2P-printing can be obtained within solid gel in any required time by i) allowing liquid form of HCC-polymers to diffuse within the pre-existing gel, ii) fabricating $3 \mathrm{D}$ hydrogel objects by using a multiphoton microscope equipped with a motorized xyz stage and a femtosecond near-infrared tightly-focused pulsed laser emission, iii) removing the 
un-crosslinked HCC-polymers via diffusion. b) Quantification of diffusion coefficient of 40 (gray) or 500 (black) kDa FITC-dextrans within Matrigel. Data are shown as mean \pm s.d. of 3 independent replicates; unequal variance Student's t-test; ${ }^{*} P<0.0213$. c) Left, representative confocal $z$-stack images of sequentially fabricated HCC-gel hydrogels (green) within the same Matrigel drop and printed at different xyz positions by using nearinfrared laser pulses through a multiphoton microscope; total $\Delta z=100 \mu \mathrm{m}$ or $50 \mu \mathrm{m}$ or $25 \mu \mathrm{m}$. Scale bar, 100 $\mu \mathrm{m}$. Middle and right, three-dimensional-volume reconstruction reveals the volumetric position of the various objects; coordinates are shown in red. d) Quantification of the minimum line width obtained using scan or freeline scanning mode, respectively scan and line scan. Data are shown as mean \pm s.d. of three independent replicates. e) Multiple $\mathrm{HCC}-\mathrm{Gel}$ structures of 3 independent replicates fabricated by near-infrared multiphoton laser pulses, showing the resolution of the multiphoton setup. $\Delta z=20 \mu \mathrm{m}$. Scale bar, $20 \mu \mathrm{m}$. f) Representative $3 \mathrm{D}$ reconstruction of 3 independent replicates of a $\mathrm{HCC}-\mathrm{Gel}$ spiral-shaped hydrogel fabricated using the free line-scan program of the multiphoton microscope. $\Delta z=30 \mu \mathrm{m}$. Coordinates and $50 \mu \mathrm{m}$ scale bar are shown. g) Quantification of the area of hydrogels sequentially fabricated at fixed laser power ( $1 \mathrm{~mW}$ ) and wavelength $(800 \mathrm{~nm})$ within the same Matrigel drop; each hydrogel series was analyzed just after photo-crosslinking (day 0 ) or at 2 days after the last 3D bioprinting. Data are shown as mean \pm s.d. of 3 independent replicates. $\mathbf{h}$ ) Young's modulus measured by atomic force microscopy of hydrogels photo-crosslinked at fixed laser power $(1 \mathrm{~mW})$ and wavelength $(800 \mathrm{~nm})$. Hydrogels were sequentially fabricated within the same Matrigel drop; each hydrogel series was analyzed just after photo-crosslinking (day 0 ) or at 2 days after the last 3D bioprinting. Data are shown as mean \pm s.d. of 3 independent replicates; multiple comparison one-way ANOVA was used; n.s., not statistically significant. 
a

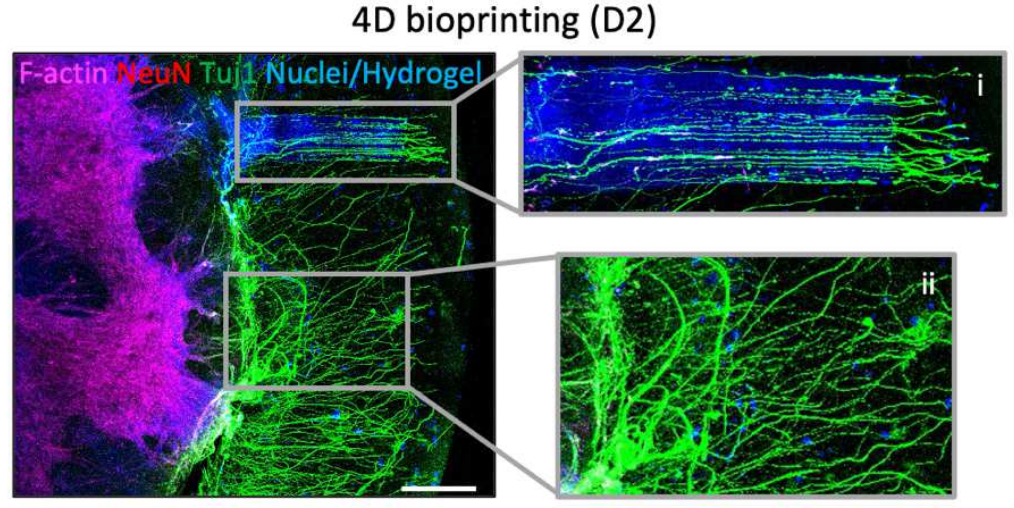

b

\section{Neural axon directionality}

$\rightarrow$ No bioprinting $\quad-$ Bioprinted Hydrogels

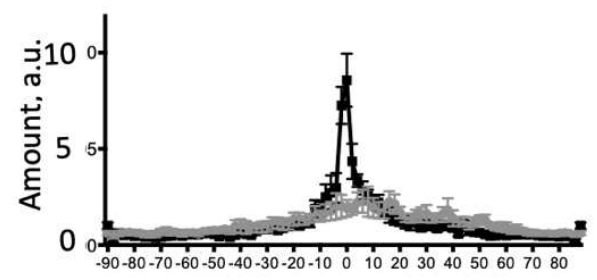

Degree
C 4D bioprinting (D1)

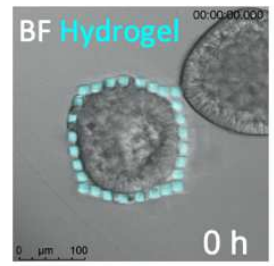

d

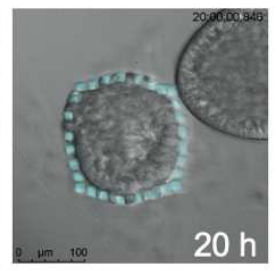

Cancer cell migration
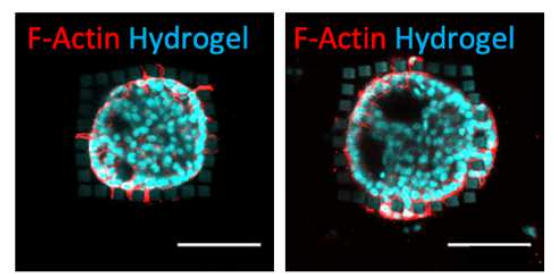

e

4D bioprinting (D1)
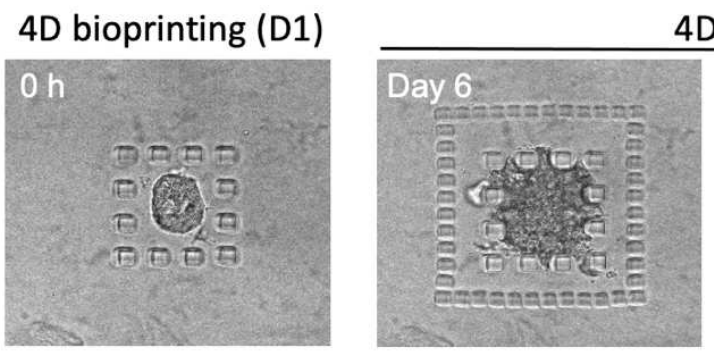

4D additive bioprinting (D7)
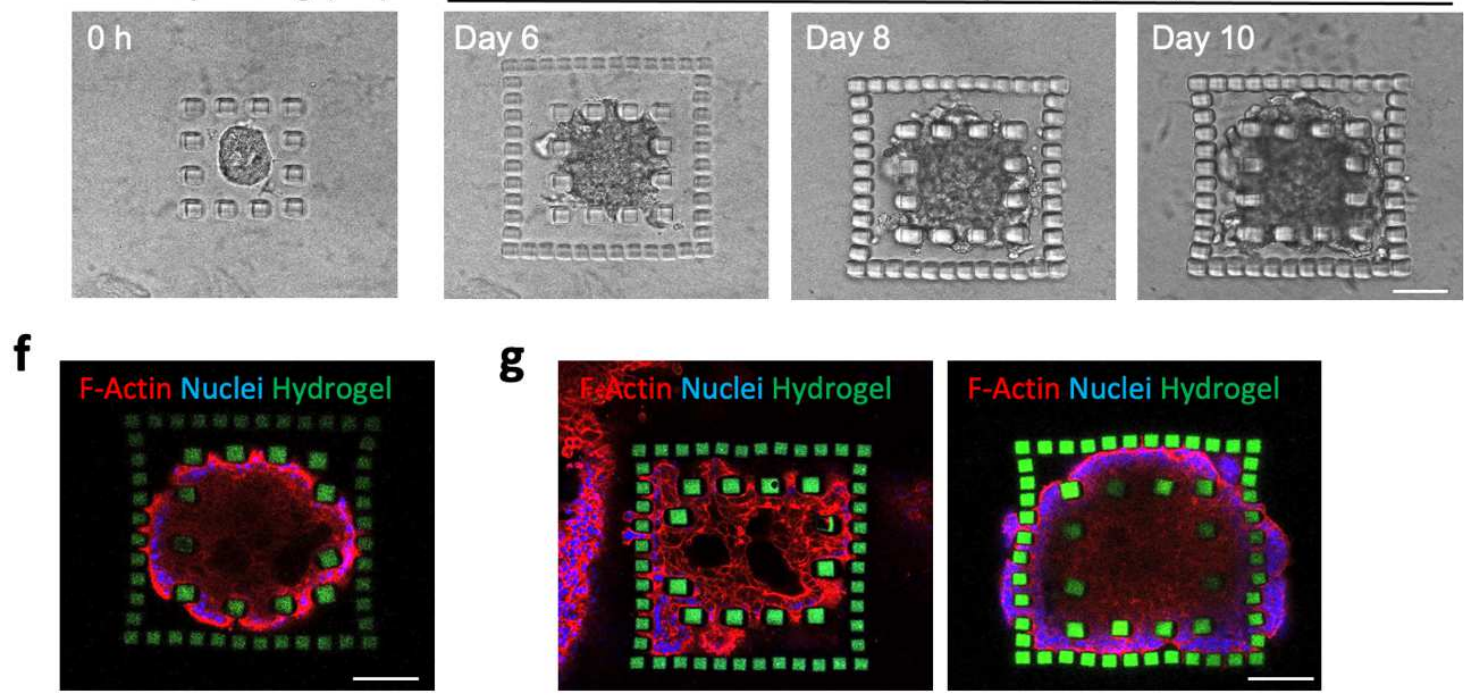

Figure 2.

Temporal control of organ-like and organoid 3D cultures by dynamic 4D live bioprinting of hydrogelin-hydrogel. a) Representative fluorescence images of a spinal cord culture showing alignment of axons protruding within fabricated hydrogel (i) as opposed to randomly oriented axon organization in absence of the hydrogel (ii). Scale bar, $200 \mu \mathrm{m}$. b) Quantification of neural projection directionality performed in area where the neural projections were far (no bioprinting) or in proximity (bioprinted hydrogels) of the fabricated hydrogelin-hydrogel structures. c) Brightfield time lap images (0, 8, 20 hours) of a hydrogel-embedded tumor spheroid (day 6 post printing) growing within a cage of HCC-PEG pillars fabricated 1 day of organoid culture. Scale bar, $100 \mu \mathrm{m}$. d) Fluorescent images of the tumor spheroid in $\mathbf{c}$, showing different stages of cellular migration through

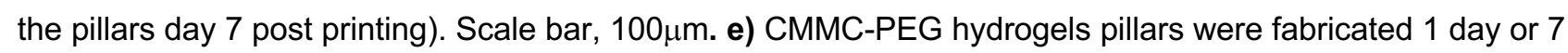
days of organoid culture (days 0 and 6 post printing, respectively) around a hydrogel-embedded growing tumor organoid. The brightfield images show the growth of the caged tumor spheroid at different time points. Scale 
bar, $100 \mu \mathrm{m}$. f) Representative fluorescent image of tumor spheroid as in $\mathbf{e}$ at 14 days from first bioprinting step (day 15 of organoid culture). Scale bar, $100 \mu \mathrm{m}$. g) Representative fluorescent images of cancer cells protruding through the bars of the first bioprinted hydrogel cage, invading the surrounding space and migrating through the pillars of the second fabrication step. Scale bar, $100 \mu \mathrm{m}$. 
a
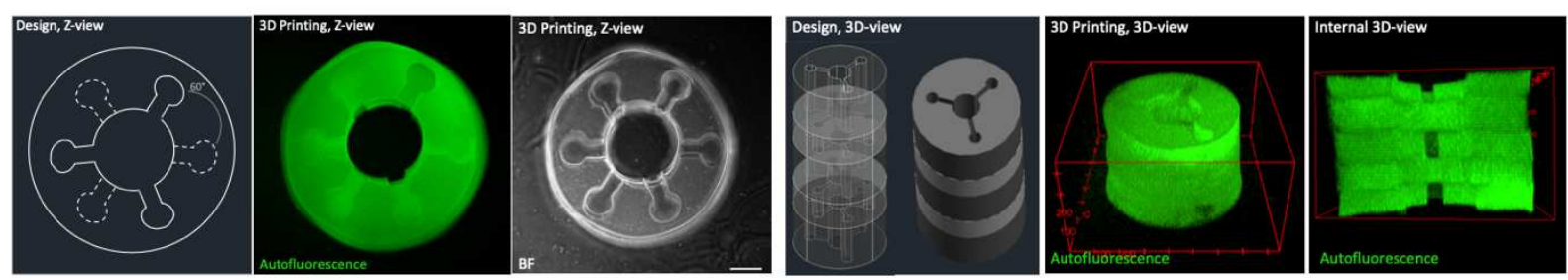

b
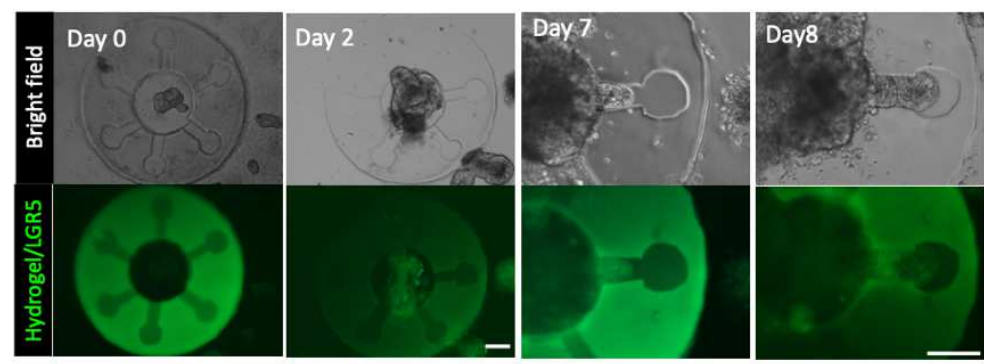

d
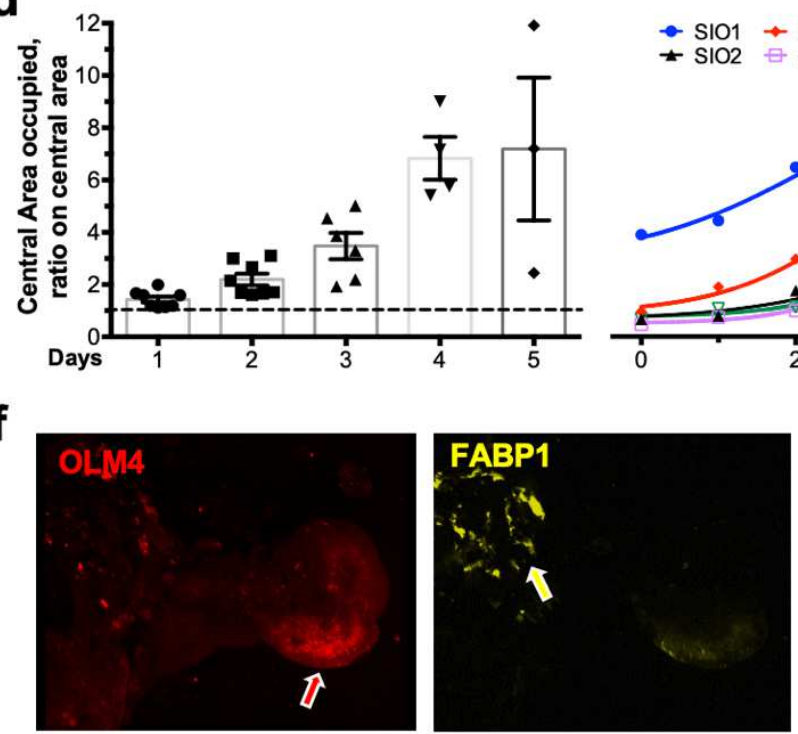

c

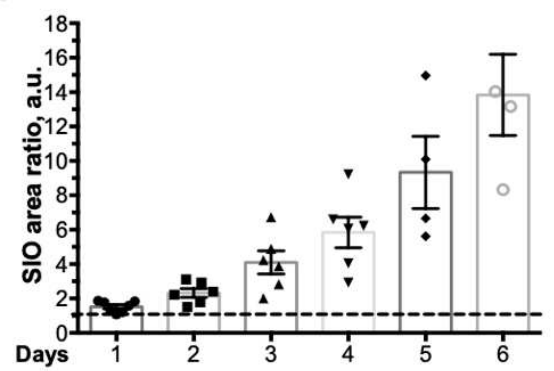

e f

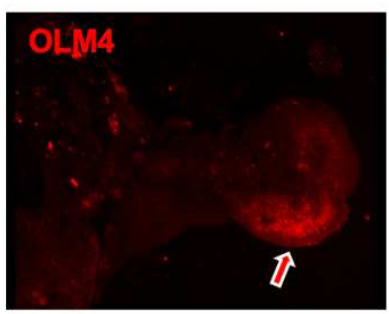

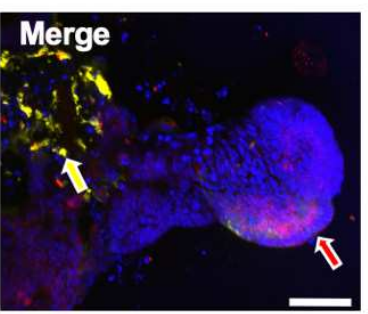
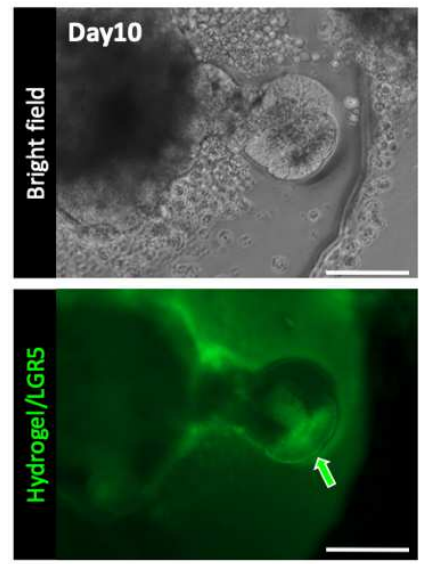

Figure 3.

Supra-organoid driven intestinal organoid morphogenesis via hydrogel-in-hydrogel live bioprinting. a) Representative bright field and fluorescence images showing $60^{\circ}$ primordial small intestine design and HCCgel hydrogels (left panel, top view; right panel, 3D reconstruction view). Scale bar $100 \mu \mathrm{m}$. b) Representative bright field (upper) and fluorescence (lower) images of mSIOs just after primordial small intestine HCC-gel hydrogel printing or 2, 7 or 8 days of culture and 4D bioprinting. Budding was observed according to the defied shape of the hydrogel. Scale bar $100 \mu \mathrm{m}$. c) Quantification of the ratio between the area of the organoid at day 0 of culture (dashed line) and the area of the organoid during the following culture days (1-6 days) for the overall. Statistical analysis is shown in Suppl. Table 1. d) Left, ratio between the central area of the primordial intestine-shaped hydrogels the organoid at seeding time (dashed line) and the area occupied by the organoid from day 1-6 of culture. Statistical analysis is shown in Suppl. Table 2. Right, Quantification of the percentage of central area occupied by the mSIOs during the culture (0-6 days). Calculation of the percentage was shown for 5 independent mSIO cultures. e) Representative bright field (upper) and fluorescence (lower) images showing mSIO budding after 10 days of culture within the primordial small intestine-shaped HCC-gel hydrogel. The arrow points at the LGR5 (green) cells. Scale bar $100 \mu \mathrm{m}$. f) Representative images showing 
immunofluorescence analysis for OLM4 (red) and FABP1 (yellow) of mSIO cultured for 10 days within the primordial small intestine-shaped HCC-gel hydrogel. Nuclei are stained with Hoechst (blue). The arrows point at the branched (red) or central (yellow) portion of the mSIO in respect to the hydrogel. Scale bar $50 \mu \mathrm{m}$. 
a
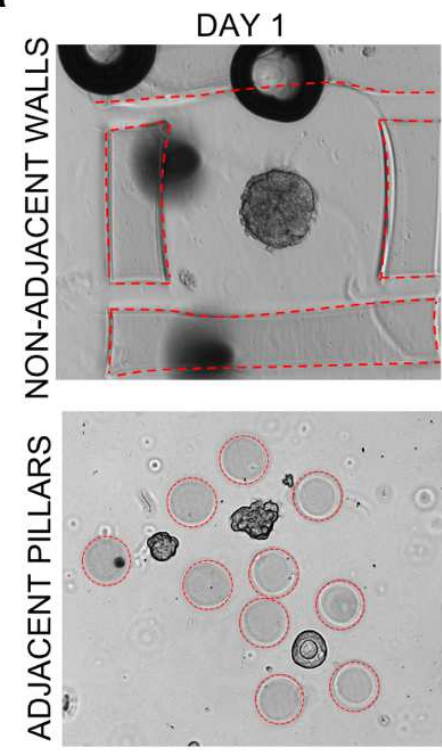

b
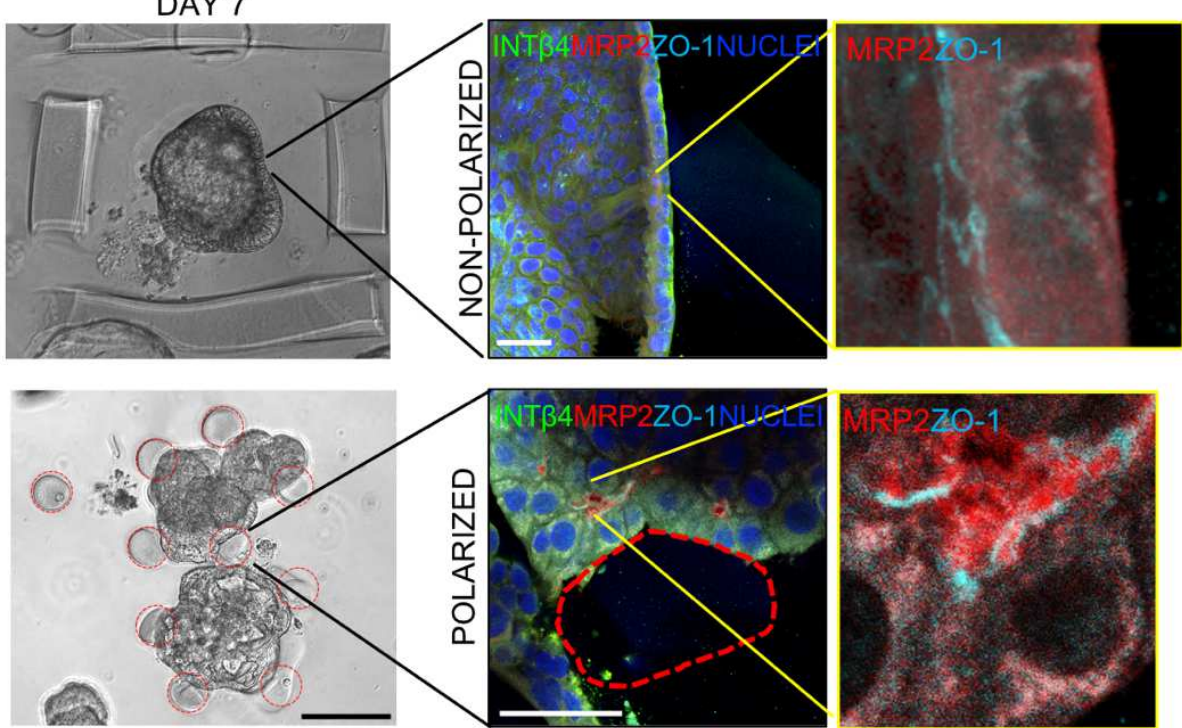

C

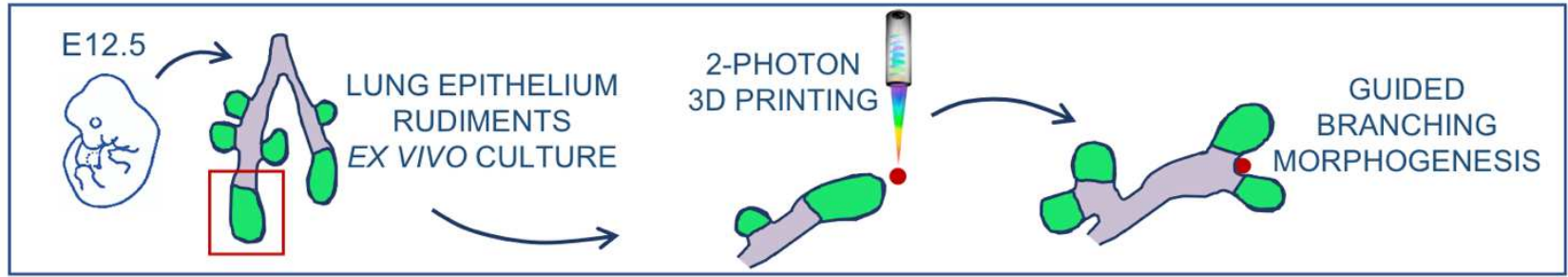

d

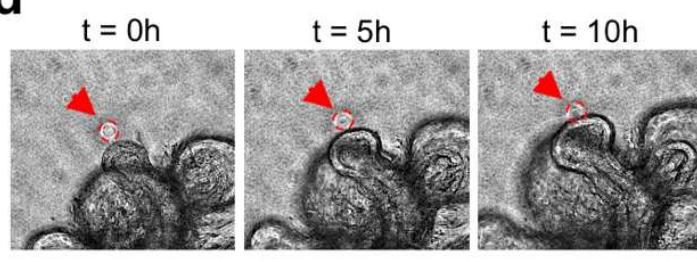

e

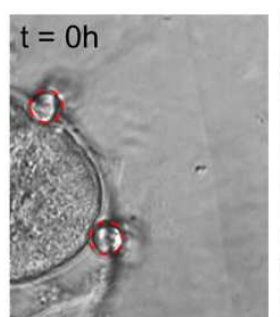

f

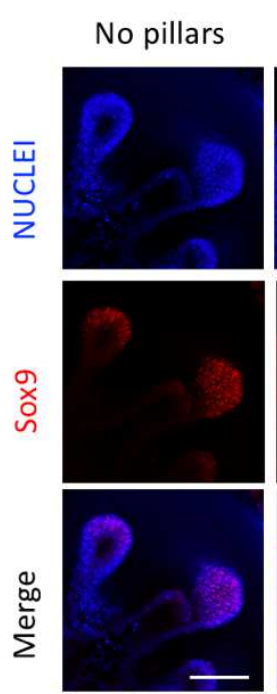

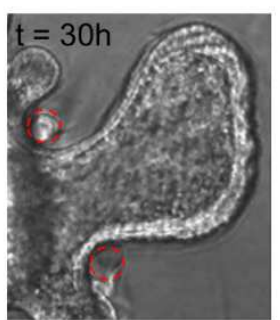

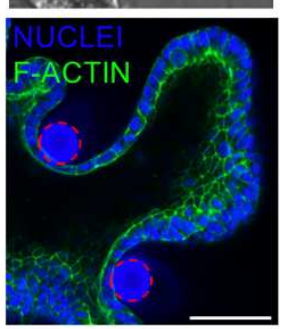

$t=20 h$

$\mathrm{t}=25 \mathrm{~h}$

$\mathrm{t}=30 \mathrm{~h}$

\section{the $30 \mathrm{~h}$}
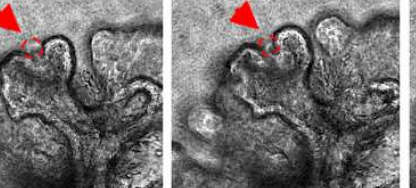

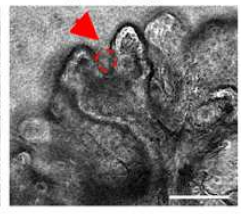

$\mathrm{t}=15 \mathrm{~h}$

With pillars
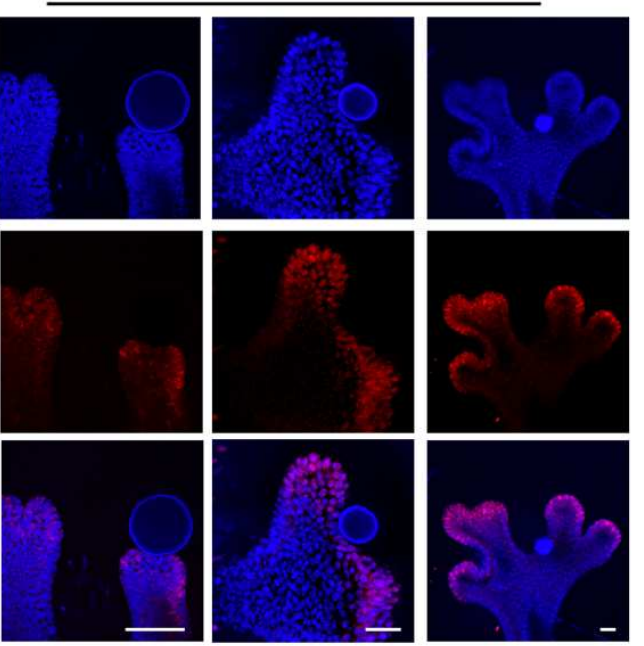

Figure 4.

3D geometrical constrains imposed by hydrogel-in-hydrogel live bioprinting on organpoid and organotypic cultures. a,b) Induction of polarization in human fetal hepatocyte organoids. a) Bright field images showing two printing strategies: distant walls not touching the growing organoid (above) and adjacent 
pillars touching the growing organoids (below) after 7 days of culture. Scaler bar $100 \mu \mathrm{m}$. b) Immunofluorescent panels showing non-polarized organoid distant from the printed structures (above) and a polarized organoid in correspondence of the printed pillars (below). Integrin beta-4 (INTß4) shown in green, multidrug resistanceassociated protein 2 (MRP2) shown in red, zonula occludens-1 (ZO-1) shown in cyan. Nuclei are stained with Hoechst (blue). Scale bars $50 \mu \mathrm{m}$. c-f) Ex vivo culture of mesenchyme-free lung epithelium rudiments, isolated from embryonic mice at stage E12.5. c) Schematic of isolated fetal lung tips ex vivo culture and guided branching morphogenesis following 2P bioprinting of gelatin pillars (red circles) in Matrigel. d) 5-hours interval snapshots of time-lapse reconstruction of budding lung tip during guided morphogenesis around pillar (red circle, indicated by red arrow). See full time-lapse in Supplementary Video 3. Scale bar $150 \mu \mathrm{m}$. e) Bright-field images and immunofluorescent panel showing lung tip branching in between two pillars (red circles) and inner (luminal) polarity maintained (F-actin, green). Nuclei are stained with Hoechst (blue). Scale bar $100 \mu m$. f) Immunofluorescent panel showing lung tip branching in between pillar (red circle) with downregulation of sox9 (red) in correspondence of the pillar. Nuclei are stained with Hoechst (blue). Scale bars $50 \mu \mathrm{m}$. 


\section{Supplementary Files}

This is a list of supplementary files associated with this preprint. Click to download.

- Suppl.VideoS3.gif

- Suppl.videoS2.gif

- Suppl.VideoS1.gif

- SupplementaryMaterialNatMethv2.pdf 